Lasierra, G.; Carreras, D.; Montoya, M. y Planas, A. (2020). The Observation in Context of Level Actions in Team Handball. Revista Internacional de Medicina y Ciencias de la Actividad Física y $\begin{array}{llllll}\text { el Deporte } & \text { vol. } & 20 & (79) & \text { pp. } & 435-451\end{array}$ Http://cdeporte.rediris.es/revista/revista79/artobservacion1171.htm

DOI: http://doi.org/10.15366/rimcafd2020.79.004

\title{
ORIGINAL
}

\section{LA OBSERVACIÓN EN CONTEXTO DE LOS NIVELES DE ACCIÓN EN BALONMANO}

\section{THE OBSERVATION IN CONTEXT OF LEVEL ACTIONS IN TEAM HANDBALL}

\author{
Lasierra, G. ${ }^{1}$; Carreras, D. ${ }^{1}$; Montoya, M. ${ }^{2}$ y Planas, A. ${ }^{3}$ \\ ${ }^{1}$ Profesor titular, INEFC - Universitat de Lleida (España) glasierra@inefc.es, dcarreras@inefc.es \\ 2 Entrenador, Qatar Handball Association (Qatar) manolomontoya9@gmail.com \\ ${ }^{3}$ Catedrático, INEFC - Universitat de Lleida (España) aplanas@inefc.es
}

Código UNESCO / UNESCO code: 5899 Otras especialidades pedagógicas (Educación Física y Deporte) / Other pedagogical specialities (Physical Education and Sports).

Clasificación Consejo de Europa / Council of Europe Classification: 4 Educación Física y deporte comparado / Physical education and sport compared.

Recibido 26 de agosto de 2018 Received August 26, 2018

Aceptado 18 de abril de 2020 Accepted April 18, 2020

\section{RESUMEN}

Esta investigación plantea como objetivo principal diseñar un instrumento de registro que permita describir y relacionar tres niveles de acción del balonmano (juego de equipo, juego grupal y juego individual), vinculándolos al contexto de competición y atendiendo a su eficacia. Siguiendo las directrices de la metodología observacional se opta por un sistema combinado de formatos de campo y sistema de categorías, utilizando un diseño de investigación de carácter ideográfico, puntual y multidimensional. Se utiliza como instrumento de registro el software Dartfish TeamPro V.4.5 para analizar siete partidos de categoría absoluta, y siete partidos de categoría infantil, generándose datos de tipo concurrente y de evento. Se aplican técnicas estadísticas descriptivas e inferenciales bivariadas para el análisis relacional de los datos. Destacamos los resultados que constatan la asociación entre sistemas de juego y procedimientos colectivos tanto ofensivos como defensivos, y la dificultad en establecer relaciones significativas entre el ataque y la defensa.

PALABRAS CLAVE: Balonmano, contexto, sistema, procedimiento, acción, eficacia. 


\section{ABSTRACT}

The main aim of this research was to design a recording tool that allows describing and relating three levels of action in team handball (team play, group play and individual play), linking them to the competition context and taking their effectiveness into account. Following the guidelines of observational methodology, we selected a combined system of field formats and system of categories, using an ideographic, specific and multidimensional research design. Dartfish TeamPro V.4.5 software, was used as a recording instrument to analyse seven matches in the top category, and seven matches in the children's category, generating concurrent and event data. Bivariate descriptive and inferential statistical techniques were used for the relational analysis of the data. The results confirmed the association between game systems and collective procedures, both offensive and defensive, as well as the difficulty to establish significant relationships between attack and defense.

KEY WORDS: Team handball, context, system, procedure, action, effectiveness.

\section{INTRODUCCIÓN}

Existen distintas aproximaciones al análisis de los deportes desde una perspectiva sistémica. A disciplinas como la praxiología (Parlebas, 2008) y los modelos horizontales estructurales (Bayer, 1986), que analizan el deporte como estructura y función en relación a un contexto, se añaden aportaciones de disciplinas que analizan el sistema deportista y / o equipo: las teorías de aprendizaje no lineal (Chow, Davids, Button, y Renshaw, 2016), los sistemas complejos (Kelso, 2014) y la psicología ecológica (Araújo, Ripoll, y Raab, 2009), todas ellas centradas en la relación indivisible sujeto - entorno.

Integrando las diferentes propuestas, podemos dibujar un modelo de los deportes de equipo en el que el contexto sea el foco central de observación, que permita constatar las relaciones sistémicas pertinentes desde los equipos y jugadores enfrentados (como sistemas) hacia el deporte (transferencia), y desde el deporte (como sistema) hacia los equipos enfrentados (información) (Lasierra, 2017). El contexto se concebiría como la aplicación de un conocimiento (SerraOlivares y García-López, 2016), en situaciones con cambios constantes (González-Villora, García-López, y Contreras-Jordán, 2015).

Siguiendo las directrices de Lago (2000) se establecen tres niveles de aproximación al desarrollo de las acciones de juego: las acciones de juego de equipo (sistemas de juego), las acciones de juego grupales (procedimientos colectivos de juego) y las acciones de juego individuales.

Los factores contextuales parten de la noción de contexto como escenario (Jiménez Jiménez, 2003) y se refieren a la coincidencia, en el mismo, de determinada disposición de los elementos estructurales del balonmano y de la 
diferente interpretación de modelos de juego de los equipos y de las capacidades de los jugadores, que modifican la dinámica de funcionamiento de juego. A su vez consideramos que el rendimiento, el resultado y la eficacia, no forman parte de los factores contextuales, sino que son consecuencia de la competencia mostrada por jugadores y equipos en cada una de las situaciones de juego planteadas. Sintetizamos nuestra visión en la figura 1.

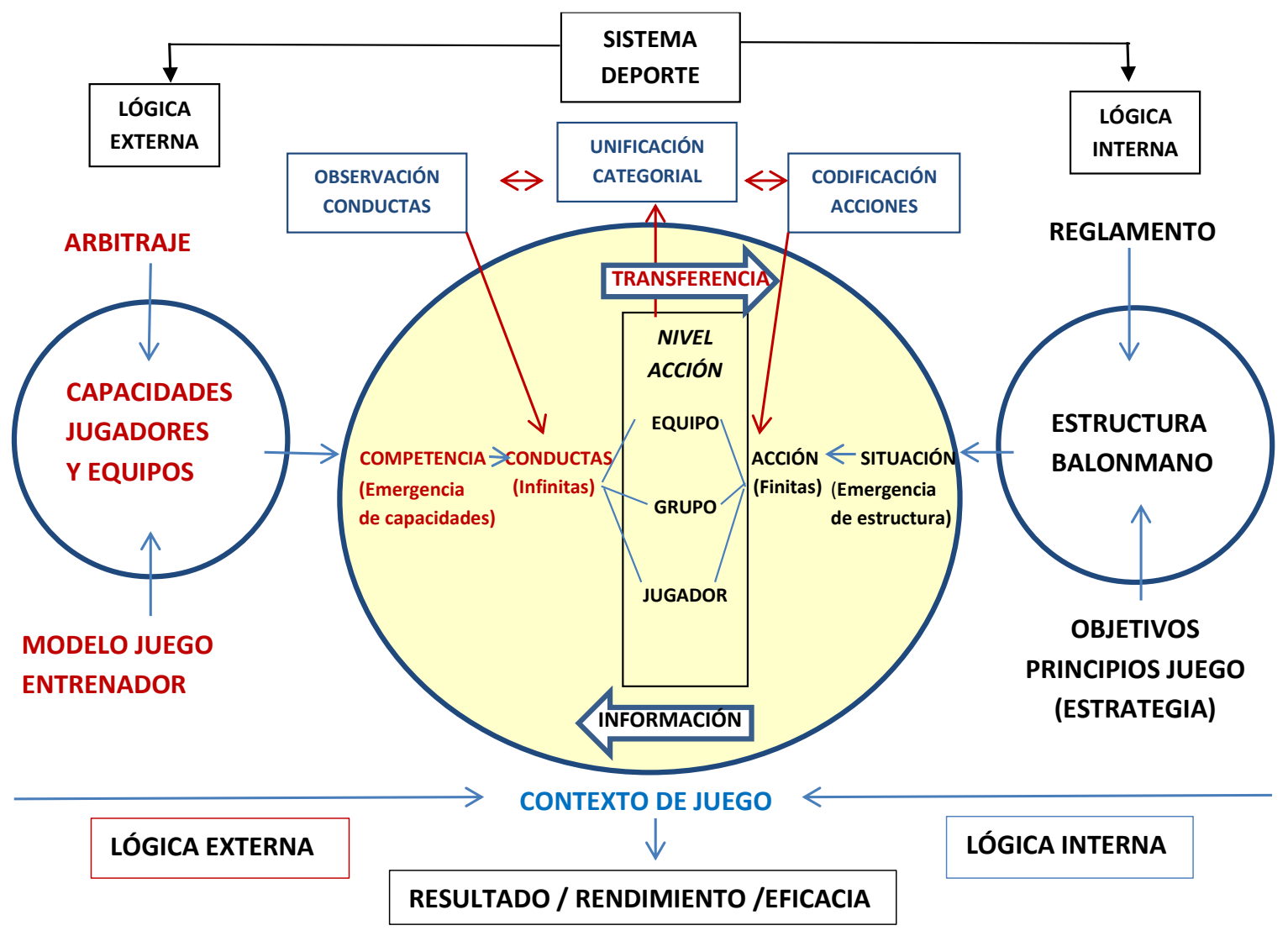

Figura 1. La observación de acciones y conductas en contexto de juego.

\section{MÉTODO}

Seleccionamos el Método Observacional (MO) (Anguera y Hernández Mendo, 2015) como método de investigación, al disponer de diseños de investigación que comparten con nuestra finalidad las siguientes características: estudio ideográfico, transducción de la realidad, holismo, unidad de observación molar, bajo nivel de control ambiental y de la muestra, alto nivel de control del observador, y análisis de datos cualitativo-cuantitativo.

\subsection{OBJETIVOS}

El objetivo principal es diseñar un instrumento de registro que permita describir y relacionar tres niveles de acción del balonmano (juego de equipo, juego grupal y juego individual), vinculándolos al contexto de competición y atendiendo a su eficacia. El objetivo secundario es aplicar un mismo diseño a la observación de dos categorías diferentes de competición (absoluta e infantil). 


\subsection{PARTICIPANTES}

En nuestro trabajo empírico tomamos como muestra a ocho equipos masculinos de categoría absoluta (ABS), participantes en la Copa del Rey 2012, así como a ocho equipos masculinos de categoría infantil (INF), participantes en el Campeonato de España de Clubes 2012 (jugadores de 12 a 14 años).

\subsection{INSTRUMENTO DE OBSERVACIÓN}

Se aplicó un sistema ad hoc combinado de formatos de campo y sistema de categorías, recomendado para estudios multidimensionales por la MO (Anguera, Blanco-Villaseñor, Losada, y Hernández Mendo, 2000).

Se propusieron para nuestro estudio cinco macrocriterios que se desglosaron en los criterios y categorías que se indican en la tabla 1.

Marco situacional (excluido en la tabla): incluyó cinco criterios cambiantes que nos ayudaron a contextualizar las diferentes variables que puede aparecen durante el juego. Los criterios fueron: equipo observado (EQ), período de juego (PAR), diferencia en el marcador (DM), fase del juego ofensivo de transición (TRA, TRC), fase del juego ofensivo posicional (AP), y situación numérica (SN).

Marco estratégico (sistemas de juego): comprendió seis criterios que en su totalidad describían la estructura y la dinámica en los cambios que se producen en los sistemas de juego, entre y durante las diferentes secuencias de ataque posicional: juego ofensivo inicial (JOI), transformación del juego ofensivo (TJO), juego ofensivo final (JOF), juego defensivo inicial (JDI), transformación del juego defensivo (TJD), y juego defensivo final (JDF).

Marco estratégico (procedimientos colectivos): comprendió cuatro criterios que en su totalidad describían la estructura y la dinámica en los cambios que se producen en los procedimientos colectivos ofensivos y defensivos en cada secuencia de juego. Los criterios seleccionados fueron: procedimientos colectivos con balón (PCO), procedimientos colectivos sin balón (PCSB), puesto específico del jugador colaborador sin balón (A, B, C, D, E, F), y procedimientos colectivos defensivos (PCD).

Marco estratégico (acciones individuales): comprendió cinco criterios que en su totalidad describían los cambios que se producían en las acciones individuales ofensivas y defensivas de juego. Se consideraron los siguientes criterios: preparación del ataque (FPR), indicador de inicio del ataque definitivo (FII), puesto específico del jugador con balón ( $A, B, C, D, E, F)$, zona de finalización con balón (ZFCB) y zona del defensor próximo (ZDP).

Marco de la eficacia: incluyó siete criterios que en su totalidad describían el resultado de cualquiera de las situaciones de juego ofensivo observadas en un partido, que determinarían posteriormente la eficacia de ataque y de lanzamiento. Los criterios seleccionados fueron: error reglamentario (RER), error 
técnico-táctico o pérdida de balón (RET), error de lanzamiento (REL), acierto del ataque (RAA), acierto de lanzamiento (RAL), resultados de la transición (RTR), y resultados intermedios (RIN). 
Rev.int.med.cienc.act.fís.deporte - vol. 20 - número 79 - ISSN: 1577-0354

Tabla 1. Criterios y categorías del marco estratégico (niveles acción) y del marco eficacia.

\begin{tabular}{|c|c|c|c|c|}
\hline $\begin{array}{c}\text { MARCO (Marco } \\
\text { situacional no incluido) }\end{array}$ & CRITERIOS & CÓD. CRITERIO & CATEGORÍA & CÓD. CATEGORÍA \\
\hline \multirow{19}{*}{ SISTEMAS DE JUEGO } & \multirow{5}{*}{ Juego ofensivo Inicial } & \multirow[t]{5}{*}{$\mathrm{JOI}$} & 3:3 1 pivote zona & $\mathrm{JOI331}$ \\
\hline & & & 3:3 2 pivotes zona & J01332 \\
\hline & & & 2:4 zona & $\mathrm{JOI24}$ \\
\hline & & & 4:2 zona & $\mathrm{JOI} 42$ \\
\hline & & & Otros: individual / mixta & JOIIN / JOIMI \\
\hline & \multirow{3}{*}{$\begin{array}{l}\text { Transformación del juego } \\
\text { ofensivo }\end{array}$} & \multirow[t]{3}{*}{ TJO } & Transform. 1á Línea & TJO1L \\
\hline & & & Transform. 2a Línea & $\mathrm{TJO} 2 \mathrm{~L}$ \\
\hline & & & \begin{tabular}{|l|l} 
Transf. 1ay ya Línea \\
\end{tabular} & TJOT2 \\
\hline & Juego ofensivo final & JOF & \multicolumn{2}{|c|}{ Mismas categorías que JOI } \\
\hline & \multirow{6}{*}{ Juego defensivo Inicial } & \multirow[t]{6}{*}{ JDI } & \begin{tabular}{|l|} 
Defensa 3:3 \\
\end{tabular} & JDI33 \\
\hline & & & \begin{tabular}{|l|} 
Defensa 4:2 \\
\end{tabular} & JDI42 \\
\hline & & & Defensa 3:2:1 & JDI321 \\
\hline & & & Defensa 6:0 & JDI60 \\
\hline & & & \begin{tabular}{|l|} 
Defensa 5:1 \\
\end{tabular} & JDI51 \\
\hline & & & Otros: individual/mixta & JDIIN/JDIMI \\
\hline & \multirow{3}{*}{$\begin{array}{l}\text { Transformación del juego } \\
\text { defensivo }\end{array}$} & TJD & Transformación 1a a 2a Línea & TJD12 \\
\hline & & & Transformación 2a a 1a Línea & TJD21 \\
\hline & & & Transformación misma línea & TJDMI \\
\hline & Juego defensivo Final & JDF & $\begin{array}{r}\text { Mismas categoría } \\
\end{array}$ & as que JDI \\
\hline & & PCO1 & \begin{tabular}{|l|} 
Puntos de apoyo \\
\end{tabular} & PAPO \\
\hline & & & Pase y va & PAVA \\
\hline & & & Pentraciones sucesivas & PPSS \\
\hline & Procedimientos colectivos & & Repetición Pase & RRPP \\
\hline & ofensivos 1 (numeración en & & Cruce & CRUC \\
\hline & función de la ocurrencia de & & Permuta & PERM \\
\hline & PCO en una secuencia) & & Fijación / Movilización & FIJA \\
\hline & & & Cortina & CORT \\
\hline & & & Bloqueo & BLOQ \\
\hline & & & Pantalla & PANT \\
\hline & & PCD1 & \begin{tabular}{|l|l} 
Deslizamiento \\
\end{tabular} & DESL \\
\hline H & Procedimientos colectivos & & Cobertura/ayuda preventiva & COBE \\
\hline & defensivos 1 (numeración & & \begin{tabular}{|l|} 
Ayuda decisiva \\
\end{tabular} & ADEC \\
\hline & den función de la ocurrencia & & Cambio oponente misma lín & COPM \\
\hline & den función de la ocurrencia & & Cambio oponente distinta lín & COPD \\
\hline & & & Doblaje & DOBL \\
\hline & & & Blocaje colectivo & $\mathrm{BCOL}$ \\
\hline & Juga dor colaborador 1 & JCOL1 & Puesto específico & $A-B-C-D-E-F$ \\
\hline & Procedimientos colectivos & PCSB1 & Permuta & PERM \\
\hline & sin halón 1 (numeraciónen & & Fijación / movilización & FIJA \\
\hline & función de la ocurrencia de & & Cortina & CORT \\
\hline & función de la ocurrencia de & & Bloqueo & BLOQ \\
\hline & PCSB en una secuencia). & & Pantalla & PANT \\
\hline & Fase de preparación & FPR & Número de pases previos & PASE1/PASE+5 \\
\hline & Fase indicador inicio & FII & Inicio individual/colectivo & ININ/INCO \\
\hline ACCIONES INDIVIDUALES & Puesto específico Juga dor 1 & JUG1 & Puesto específico & $A-B-C-D-E-F$ \\
\hline & Zona final con balón 1 & ZFCB1 & Zonas limitadas campo & Z1 a Z13 \\
\hline & Zona defensor próximo & ZDP1 & Zonas limitadas campo & Z1 a Z13 \\
\hline & & RER & Pasos & RPASO \\
\hline & & & Dobles & RDOBL \\
\hline & & & Falta ataque & RFATA \\
\hline & Resultado final: error & & Invasión área & RINVA \\
\hline & reglamentario & & Pasivo & RPASI \\
\hline & & & Final tiempo juego & RFITI \\
\hline & & & Pies & RPIES \\
\hline & & & 3segundos & R3SEG \\
\hline & Resultado final; error téc. - & RET & Pérdida de balón & RPEBA \\
\hline & & REL & Poste & RFPOS \\
\hline & Resultado final: error & & Final periodo o partido & RFPAR \\
\hline & lanzamiento & & Fuera & RFFUE \\
\hline & & & Blocaje & RFBLO \\
\hline RESULTADOS / EFICACIA & Resultado final: acierto & RAL & Gol & RGOL \\
\hline & lanzamiento & & Gol + 2 minutos / amon. / des & RGOL+2 \\
\hline & & RAA & 7 metros & R7M \\
\hline & Resurta do mal: acterto & & 7 metros +2 minutos/amon. & R7M+2 \\
\hline & & & 2 minutos /descalificación & R2 \\
\hline & & RTR & Pasa a ataque posic. & RTAPO \\
\hline & Resultados transición & & Pasa a result. Interm. & RTINT \\
\hline & & & Pasa a result. Finales & RTFIN \\
\hline & & RIN & Golpe franco & RGFRA \\
\hline & & & Time Out árbitro & RTOUA \\
\hline & Resultados intermedios & & Time Out entrenador & RTOUE \\
\hline & Resultados intermedios & & Recuperación/rebote & RRECU \\
\hline & & & Saque banda a favor & RBAND \\
\hline & & & Golpe franco+amonestación & RGF+T \\
\hline
\end{tabular}




\subsection{INSTRUMENTO DE REGISTRO}

La estructura del instrumento de observación fue trasladada directamente al instrumento de registro, seleccionándose el programa informático Dartfish TeamPro V.4.5, dado que permite múltiples opciones de diseño.

\subsection{PROCEDIMIENTO}

Nos decantamos, en función de los diferentes tipos de diseños observacionales, por acogernos al cuadrante II (Anguera y Hernández Mendo, 2013), seleccionando un diseño ideográfico, puntual y multidimensional.

En relación al proceso y a las fases de investigación, la presente investigación se ha caracterizado por las siguientes particularidades a partir de las directrices establecidas por el MO:

a) Unidades de conducta: se registraron eventos y puntualmente estados considerados como eventos.

b) Instrumento de observación: se elaboró un sistema combinado de formatos de campo y sistema de categorías.

c) Muestreo observacional (muestreo de sujetos): la muestra de sujetos se compuso de la selección de equipos de clubes masculinos del Estado español, distribuidos en dos grupos de competición, categorías infantil y absoluta.

d) Muestreo observacional (muestreo inter e intrasesional): el muestreo intrasesional se caracterizó por ser de tipo multievento y de carácter continuo. En relación con el muestreo intersesional, el período de observación se adscribió a las fechas de desarrollo de los campeonatos observados.

e) Tipo y parámetros de registro: en base a los requisitos del $\mathrm{MO}$ y a través de un proceso de sistematización del registro, se obtuvo en la fase activa o científica un registro altamente sistematizado. Los parámetros de registro se basaron en unidades primarias de frecuencia, orden y duración.

f) Análisis de la calidad del dato y validación del instrumento:

se realizaron pruebas de validez del instrumento de observación a partir de una selección de expertos, tal y como se establece en múltiples investigaciones (Gamonales, León, Muñoz, González-Espinosa, y lbáñez, 2018; Serra-Olivares y García-López, 2016). En nuestra investigación los dos criterios exigidos eran 1) titulación académica: ser doctor o estar en su proceso de obtención y; 2) titulación deportiva: poseer el título de entrenador nacional de balonmano. Seis expertos que cumplían estos requisitos fueron los encargados de modificar y finalmente aceptar los criterios esgrimidos en un cuestionario de respuesta binaria. Asimismo, un subgrupo de tres miembros del panel de expertos colaboró 
en el proceso de obtención de la fiabilidad del instrumento, sometiéndose a los periodos de formación y de entrenamiento, así como a dos sesiones de evaluación de la fiabilidad inter-observadores, para conseguir que los resultados de concordancia fueran estadísticamente correctos, obteniéndose un Índice Kappa de Cohen de 0,985.

\subsection{ANÁLISIS DE LOS DATOS}

Los datos obtenidos pueden considerarse de tipo II, es decir, concurrentes (desde el punto de vista de su ocurrencia) y de registro de eventos (atendiendo a la base del registro). A partir del registro de las diferentes categorías, en su mayoría variables cualitativas de escala nominal, se realizó un análisis descriptivo (clasificación y organización de los datos) e inferencial (extracción de conclusiones) para cada uno de los apartados y niveles previstos (Anguera y Hernández Mendo, 2015).

Técnicas descriptivas: La organización de los resultados descriptivos, incluyó como primer paso, la realización de un recuento de las ocurrencias dadas por categorías posibles en valores de frecuencia absoluta, o bien, un porcentaje del valor de cada una de las categorías en el conjunto de categorías posibles en valores de frecuencia relativa. Posteriormente se realizaron operaciones para valorar la centralidad y/o la dispersión de los datos obtenidos.

Técnicas inferenciales de relación entre variables: El diseño de la investigación se concentró básicamente en el análisis inferencial de la comparación de variables, tanto en casos en los que se relacionaban las mismas categorías entre ABS e INF, como cuando ABS e INF eran considerados como variables distintas, pero sin la pretensión de analizarlos como grupos. En ambas circunstancias, el primer paso fue aplicar tablas de contingencia para establecer la relación entre dos o más variables cualitativas, a través de la recogida de datos expresada en frecuencias absolutas. Posteriormente se compararon los valores reales con los posibles en las tablas de contingencia, aplicando el test de ji-cuadrado $\left(\square^{2}\right)$, obteniendo valores $\mathrm{p}(p)$ que indicaban la significación estadística. El valor obtenido mediante el test ji-cuadrado proporcionó la significación de determinada relación, pero no la cantidad o el valor de relación. Para ello realizamos el cálculo de la $V$ de Cramer ( $V$ p para cuantificar el grado de relación.

\section{RESULTADOS}

En un análisis independiente de las categorías absoluta (ABS) e infantil (INF), sin intención de compararlas, se obtienen las siguientes relaciones significativas en función de las relaciones intra e intermarcos consideradas.

\subsection{MARCO SITUACIONAL Y SUS RELACIONES}

Intentos de ataque y duración: se detecta una diferencia significativa entre ABS e INF. Se aplicó la prueba $t(t)$ al criterio juego de transición, obteniéndose un valor $p(p)$ que constató una diferencia significativa entre ABS e INF $(t=4,489 ; p$ $<0,0005$; IC 95\% 0,558 a 1,425 s). Se aplicó la prueba ANOVA $(F)$ a las 
diferentes tipologías de ataque posicional ( $F=17,840 ; p<0,0005)$, constatándose una menor duración de intentos consecutivos de ataque en una misma posesión (ABS: AP1 = 11,21", AP4 = 6,33"; INF = AP1 = 7,79", AP4 = $5,2 ")$.

Participación por puestos específicos en cada secuencia: se detecta una diferencia significativa entre ABS e INF. Se valora la relación entre las categorías de los criterios puestos específicos y orden de su intervención en la secuencia de juego, y se observa un valor de intensidad de asociación débil, tanto en ABS $\left(\square^{2}=769,69 ; p<0,0005 ; V=0,092\right)$, como en INF $\left(\square \square^{2}=91,753 ; p<0,0005 ; V\right.$ $=0,044)$. Se observa que cuanto más dura la secuencia, mayor es la participación de la segunda línea ofensiva (valor máximo: $A B S$ : $E=31,8 \%$, INF: $\mathrm{D}=50 \%$ ).

\subsection{MARCO NIVELES DE ACCIÓN Y SUS RELACIONES}

Sistema de juego ofensivo final y procedimientos colectivos con balón: se detecta una diferencia significativa entre ABS e INF. Se valora la relación entre las categorías de los criterios procedimientos colectivos ofensivos y sistema de juego ofensivo final, con un valor de intensidad de asociación débil, tanto en ABS $\left(\square^{2}=49,563 ; p<0,0005 ; V=0,049\right)$, como en INF $\left(\square^{2}=45,165 ; p<0,0005 ; V=\right.$ $0,058)$. Se asocian los sistemas ofensivos impares -3:3(1) y 3:3(2)- a la activación de cruce (CRUC) $(24,5 \%$ en $A B S)$, y los sistemas ofensivos pares $2: 4$ y 4:2- a la activación de fijaciones (FIJA) (21,6\% en ABS). En INF se mantiene esta tendencia, pero con menores diferencias.

Sistema de juego defensivo final y procedimientos colectivos defensivos: se detecta una diferencia significativa entre ABS e INF. Se valora la relación entre las categorías de los criterios procedimientos colectivos defensivos y sistema de juego defensivo final, con un valor de intensidad de asociación débil, tanto en ABS $\left(\square^{2}=256,215 ; p<0,0005 ; V=0,088\right)$, como en INF $\left(\square^{2}=170,778 ; p<\right.$ $0,0005 ; V=0,084)$. Se asocia la defensa $6: 0$ a los procedimientos defensivos cambio de oponente en la misma línea (COPM) (ABS: $33 \%$, INF: 16,7 \%) y blocaje colectivo (BCOL) (ABS: $45 \%$, INF: $43 \%$ ). La defensa $5: 1$ se asocia a cambio de oponente en diferente línea (COPD) (ABS: 12,3\%, INF: 11,4\%).

Procedimientos colectivos sin balón entre ABS e INF: se detecta una diferencia significativa con menor utilización por parte de INF de procedimientos colectivos sin balón. Se valora la relación de las categorías del criterio procedimientos colectivos ofensivos sin balón entre ABS e INF, con un valor de intensidad de asociación débil $\left(\square^{2}=26,357 ; p<0,0005 ; V=0,150\right)$.

\subsection{MARCO EFICACIA Y SUS RELACIONES}

Procedimientos colectivos defensivos (el último de cada secuencia) y eficacia del ataque: se detecta una diferencia significativa entre ABS e INF. Se valora la relación entre las categorías de los criterios procedimientos colectivos defensivos y eficacia de ataque en ABS e INF, obteniéndose una relación significativa (a excepción de los errores reglamentarios en INF), y un valor de 
intensidad de asociación débil en: errores técnico-tácticos $\left(\square^{2}=45,602 ; p<\right.$ $0,0005 ; V=0,152)$, errores reglamentarios (ABS: $\square \square \square^{2}=91,777 ; p<0,0005 ; V=$ 0,217 ; INF: $\square \square^{2}=34,659 ; p>0,05 ; V=0,149$ ), errores de lanzamiento (ABS: $\square \square^{2}$ $=257,927 ; p<0,0005 ; V=0,170 ;$ INF: $\left.\square \square \square^{2}=90,828 ; p<0,0005 ; V=0,120\right)$, y aciertos de ataque (ABS: $\square \square^{2}=249,663 ; p<0,0005 ; V=0,107$; INF: $\square \square^{2}=$ $897,480 ; p<0,0005 ; V=0,253)$. Los procedimientos colectivos defensivos que más se asocian negativamente a la eficacia del ataque son el blocaje colectivo (BCOL) (ABS: 43,9\%, INF: $44 \%$ ) y el doblaje (DOBL) (ABS: $44 \%$, INF: 36,2\%). Los procedimientos colectivos defensivos que más se asocian positivamente a la eficacia del ataque son en ABS deslizamiento (DESL $=63,3 \%$ ) y ayuda decisiva $(A D E C=60,6 \%)$, siendo en INF: cambio de oponente en distinta línea $(\mathrm{COPD}=69,2 \%)$ y deslizamiento $(\mathrm{DESL}=60,6 \%)$.

Procedimientos colectivos sin balón y eficacia del ataque: se detecta una diferencia significativa entre ABS e INF. Se obtuvo una intensidad de asociación débil en los errores técnico-tácticos $\square \square^{\square}=10,544 ; p<0,0005 ; V=0,238$ ), así como los errores de lanzamiento en ABS $\left(\square \square^{2}=42,598 ; p<0,0005 ; V=0,164\right)$. Finalmente, los aciertos de ataque mostraron una relación significativa y fuerte intensidad de asociación (ABS: $\square^{2}=872,457 ; p<0,0005 ; V=1,033$, INF: $\square^{2}=$ 305,089; $p<0,0005 ; V=0,919$ ).

Eficacia de ataque y de lanzamiento en relación a puestos específicos y zonas de finalización (Figura 2): se detecta una diferencia significativa entre ABS e INF entre las zonas de lanzamiento y la eficacia de lanzamiento. Se obtuvieron relaciones significativas y un valor de intensidad de asociación débil en ABS ( $\square^{2}$ $=27,34 ; p=0,0001 ; V=0,295)$, y significativas moderadas en INF $\left(\square^{2}=49,33\right.$; $p=0,0001 ; V=0,441)$. Los resultados obtenidos constataron que existía una relación entre la distancia-verticalidad del lanzamiento y su eficacia. Así para ABS e INF la zona más eficaz fue la zona aérea central $(Z 11: A B S=82 \%$, INF = $88,4 \%)$. Se constata además una diferencia significativa respecto la eficacia de ataque en INF, categoría en la que se cometen más errores técnico - tácticos $\left(\square^{2}\right.$ $=15,271 ; p=0,0005 ; V=0,166)$. Por el contrario, en la relación puestos específicos y eficacia de lanzamiento, en INF se producen más aciertos de lanzamiento que en ABS detectándose una diferencia significativa en función de puestos específicos en ABS $\left(\square^{2}=12,5 ; p=0,029 ; V=0,198\right)$ por la mayor efectividad de lanzamiento del pivote, en relación al resto de puestos específicos. 

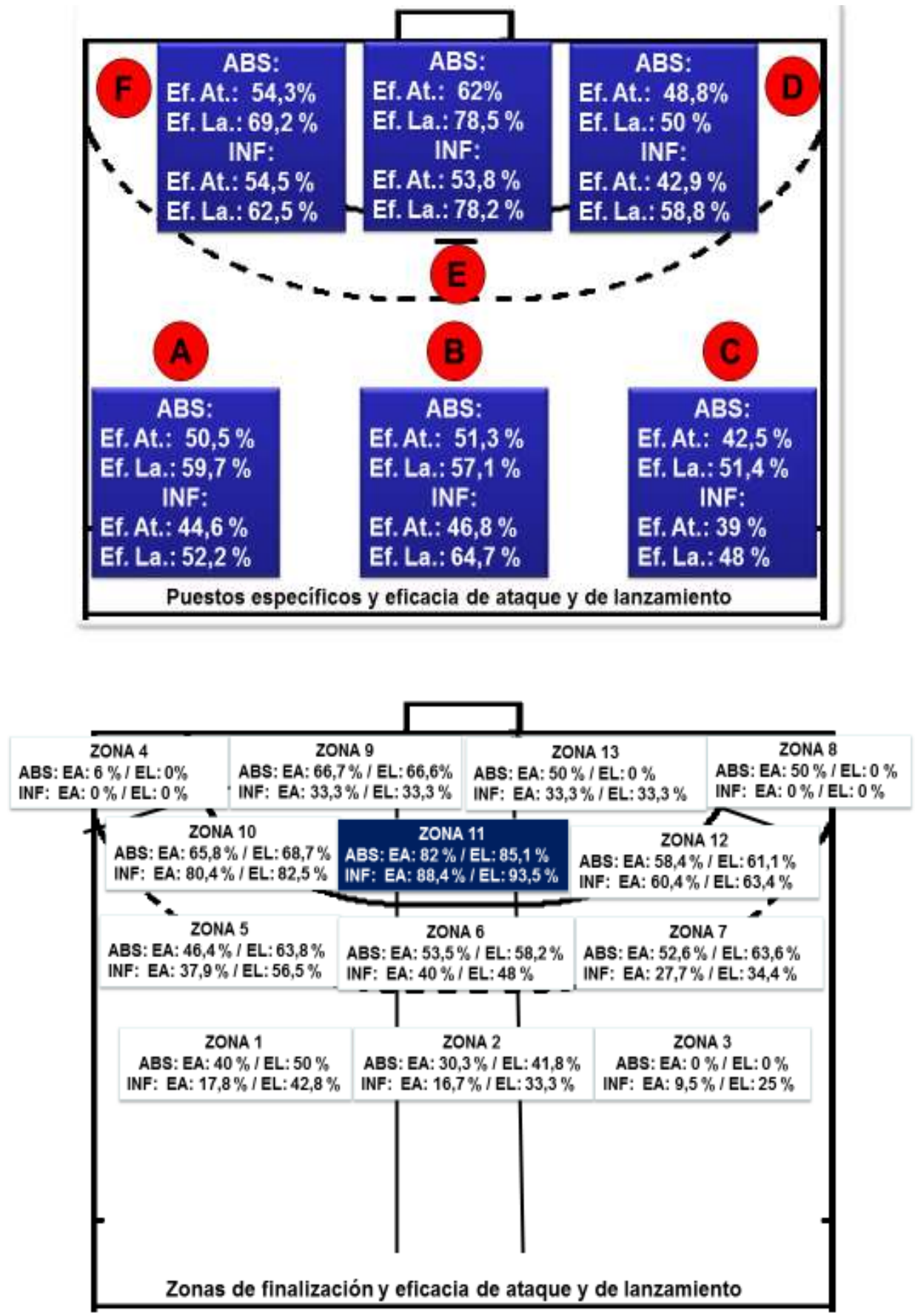

Figura 2. Puestos específicos, zonas de finalización y eficacia de ataque y lanzamiento.

\section{DISCUSIÓN}

\subsection{MARCO SITUACIONAL}

En la presente investigación se ha decidido descartar como unidad de observación tanto el concepto de posesión (Antón, 1990), como el de unidad de competición (Álvaro et al., 1995), por considerar que por su extensión temporal, no diferencian las fases del juego de forma pertinente. Por ese motivo, algunos de nuestros resultados no pueden compararse con investigaciones centradas en dichas unidades (Gutiérrez, 2006; Lozano, 2014; Montoya, 2010). 
Los resultados de este estudio, constatan una menor duración de los intentos consecutivos en una misma posesión, Estos resultados coinciden con Daza (2010). No coinciden con los resultados de Rocha Santos (2004).

Se constata que, a mayor duración de la secuencia, se produce una mayor participación de la segunda línea ofensiva. En relación a ABS, nuestros resultados coinciden con Rogulj, Srhoj, y Srhoj (2004). No ha sido posible comparar nuestros resultados con otras investigaciones en el caso de INF.

\subsection{MARCO ESTRATÉGICO DE LOS NIVELES DE ACCIÓN}

Los sistemas de juego tradicionalmente se conciben como un registro continuo de la mecánica de la acción. La descripción de los mismos se concentra en buscar unos patrones de coordinación en el propio sistema ofensivo (García Herrero et al., 2006), o defensivo (Antón, 2000) .

Consideramos que determinado sistema de juego no puede ser explicado sin considerar el del equipo contrario, por lo que en nuestro diseño intentamos constatar esas relaciones observando la dinámica real de funcionamiento y evolución de la defensa en función de las propuestas del ataque y viceversa. Sin embargo, nuestros resultados no apuntan a establecer claramente esa mutua relación ataque-defensa, por lo que, en la construcción de los sistemas de juego, en ambas categorías observadas, se constata un desarrollo del juego, que no muestra evidencias de modificarse durante determinada secuencia en función de las propuestas del equipo contrario.

Se asocian los sistemas ofensivos impares a la activación de procedimientos colectivos que definen el juego permutante mientras que los pares activan procedimientos que definen el juego posicional. Esa alta relación entre sistemas y procedimientos ofensivos coincide en rasgos generales con la investigación de Lozano (2014).

Se constata que cada uno de los sistemas de juego defensivos activa determinados procedimientos colectivos defensivos. Esa significativa relación entre sistemas y procedimientos defensivos no ha podido ser comparada.

Los procedimientos colectivos continúan definiéndose como coordinaciones grupales de movimientos que encadenados constituyen un sistema de juego. Se define un procedimiento únicamente por el desplazamiento coordinado de los jugadores intervinientes (Neves, 2004). Se construyen discursos a partir de la diferenciación de la complejidad en los procedimientos colectivos, que conlleva dificultades para establecer la línea divisoria entre procedimientos complejos y sistemas de juego (Sierra-Guzmán, Sierra-Guzmán, Sánchez, y Sánchez Sánchez, 2015). Se tiende al análisis de los procedimientos más significativos 0 al de los últimos registros de una secuencia (Sousa, Prudente, Sequeira, LópezLópez, y Hernández Mendo, 2015). 
En esta investigación se delimitan la totalidad de los procedimientos colectivos a unos mismos criterios de registro: la relación de dos jugadores con transmisión del balón (procedimientos ofensivos con balón), la colaboración de otro jugador sin balón (procedimientos colectivos sin balón), y la simultánea acción colectiva empleada en defensa entre dos jugadores (procedimientos defensivos), registrándose todos los procedimientos colectivos en cada secuencia analizada. Como resultado se observa que los procedimientos colectivos con balón más utilizados son los puntos de apoyo, apenas mencionados en la bibliografía especializada.

Se confirma una mayor utilización de procedimientos sin balón en ABS que en INF, con diferencia significativa entre ambas categorías, lo que conlleva una reflexión en relación a la teórica apuesta por defensas abiertas que deberían privilegiar el juego sin balón. Estos resultados no han podido ser comparados.

\subsection{MARCO DE LA EFICACIA}

En relación a la eficacia, consideramos adecuada la valoración de la eficacia de lanzamiento (Gutiérrez, 2006), para dar explicación al nivel individual de los jugadores a partir de la posesión como unidad de observación. Sin embargo, apuntamos que los datos de eficacia de lanzamiento, apenas aportan relaciones significativas en nuestra investigación, basada en unidades de secuencia, por lo que nos propusimos analizar también la eficacia de ataque.

Se constata que en INF se cometen más errores de ataque, pero también más aciertos de lanzamiento. Asimismo, la mayor eficacia de ataque en ABS se asocia a la mayor ocurrencia de resultados intermedios, es decir al incremento de interrupciones de secuencias ofensivas con el objetivo de mantener la posesión del balón. No ha sido posible comparar estos resultados.

Se constata que la eficacia del ataque no depende del juego con o sin transformación. En INF se muestran diferencias favorables a la eficacia de lanzamiento con transformación y $\mathrm{ABS}$ es más eficaz lanzando sin transformación, resultados que coinciden con los de Ávila Moreno (2003).

Existen otros criterios dentro de la eficacia del ataque que consideramos más representativos para asociar el éxito o fracaso a determinados sistemas de juego. Sirvan como ejemplo, por haberse obtenido diferencias significativas en nuestro estudio, los siguientes:1) zona de lanzamiento (más central y con mayor ángulo) y; 2) número de errores reglamentarios y técnico-tácticos en relación a los aciertos de ataque.

Respecto la valoración de la eficacia del ataque en la aplicación de los procedimientos colectivos, y debido también a la obtención de diferencias significativas en nuestra investigación, sugerimos atender a indicadores de los procedimientos defensivos que: 1) se vinculen con el éxito defensivo al mantener una situación de equilibrio defensivo: blocaje y doblaje y; 2) se vinculen con el éxito ofensivo al obtener el ataque un desequilibrio defensivo: ayuda decisiva y deslizamiento. 
Destacamos finalmente la relación significativa obtenida en esta investigación tanto en ABS como en INF, en el uso de procedimientos colectivos sin balón asociados a una mayor eficacia de ataque, respecto la no aplicación de los mismos, resultados que no han podido ser comparados con otros estudios.

\section{LIMITACIONES, APLICACIONES PRÁCTICAS Y CONCLUSIONES}

La limitación fundamental de esta investigación, ha sido la dificultad en poder comparar nuestros resultados con estudios previos aplicados a las etapas de formación deportiva, aspecto que pone en valor la difusión de trabajos que aporten datos relevantes en las etapas iniciales de formación de deportistas.

A partir del modelo planteado en esta investigación, se ha realizado una experiencia práctica de evaluación en deportes colectivos a través de juegos reducidos (balonmano, baloncesto y voleibol), con estudiantes universitarios del INEFC-Lleida durante el curso académico 2018-2019. El estudio ha sido presentado en el marco del 30th FIEP World Congress (Lasierra, Serna, Salas y López-Ros, 2019).

Considerando las propuestas de otros autores (López-Herrero y Arias-Estero, 2019; Morales-Belando y Arias-Estero, 2015; Pérez et al., 2019), y atendiendo a las futuras líneas de investigación desarrolladas en nuestra experiencia práctica, pretendemos validar y aplicar nuestro instrumento a la evaluación de la competencia motriz específica en deportes de equipo en centros educativos de primaria (evaluación del juego deportivo) y secundaria (evaluación de modalidades específicas), con diferentes niveles de concreción del instrumento de registro, en función del nivel educativo.

Como conclusiones de la presente investigación, destacamos:

En relación al objetivo principal: ha sido posible validar y aplicar, un instrumento de registro que conjuga los tres niveles de acción existentes en deportes colectivos y que los relaciona con el marco de la eficacia, en situaciones de competición deportiva en balonmano.

En relación al objetivo secundario: se han establecido relaciones significativas y no significativas, en el análisis comparativo entre categorías de competición en el alto rendimiento y en etapas de formación, a partir de un único instrumento de observación, que constata la utilidad y transferibilidad del mismo.

\section{REFERENCIAS BIBLIOGRÁFICAS}

Álvaro, J., González Badillo, J. J., González, J. L., Navarro, F., Molina, J. J., Portoles, J., y Sánchez, F. (1995). Modelo de análisis de los deportes colectivos basados en el rendimiento en competición. INFOCOES, 1(0), 2141.

Anguera, M. T., Blanco-Villaseñor, A., Losada, J. L., y Hernández Mendo, A. (2000). La metodología observacional en el deporte: conceptos básicos. 
Lecturas: Educación Física y Deportes, 5(24).

Anguera, M. T., y Hernández Mendo, A. (2013). La metodología observacional en el ámbito del deporte. E-balonmano.com: Revista de Ciencias del Deporte, 9(3), 135-160.

Anguera, M. T., y Hernández Mendo, A. (2015). Técnicas de análisis en estudios observacionales en ciencias del deporte. Cuadernos de psicología del deporte, 15(1), 13-30. https://doi.org/10.4321/S1578-84232015000100002

Antón, J. L. (1990). Balonmano. Fundamentos y etapas de aprendizaje. Madrid: Gymnos.

Antón, J. L. (2000). Balonmano. Perfeccionamiento e investigación. Perfeccionamiento e investigación. Barcelona: Inde.

Araújo, D., Ripoll, H., y Raab, M. (2009). Perspectives on cognition and action in sport. (D. Araújo, H. Ripoll, \& M. Raab, Eds.). New York: Nova Science Publishers, Inc.

Ávila Moreno, F. M. (2003). Aplicación de un sistema observacional para el análisis del lanzamiento en balonmano en el Mundial de Francia 2001. Apunts: Educación física y deportes, (71), 100-109.

Bayer, C. (1986). La enseñanza de los juegos deportivos colectivos. Barcelona: Hispano-Europea.

Chow, J. Y., Davids, K., Button, C., y Renshaw, I. (2016). Nonlinear pedagogy in skill acquisition. An introduction. Abingdon, Oxon: Routledge. https://doi.org/10.4324/9781315813042

Daza, G. (2010). Las habilidades del pivote en la alta competición de balonmano (Tesis doctoral). Universitat de Barcelona.

Gamonales, J. M., León, K., Muñoz, J., González-Espinosa, S., y lbáñez, S. J. (2018). Validación del IOLF5C para la eficacia del lanzamiento en fútbol para ciegos. Revista internacional de medicina y ciencias de la actividad física y deporte, 18(70), 361-381. https://doi.org/10.15366/rimcafd2018.70.010

García Herrero, J. A., Aniz, I., Barbado, F. D., Arellano, J. I., Nogales, J. F., y Blázquez, M. (2006). Análisis de los parámetros espaciales en el juego de ataque de los equipos finalistas del Campeonato del Mundo de balonmano de Portugal. Motricidad. European Journal of Human Movement, 17, 111121.

González-Villora, S., García-López, L. M., y Contreras-Jordán, O. R. (2015). Evolución de la toma de decisiones y la habilidad técnica en fútbol. Revista internacional de medicina y ciencias de la actividad física y deporte, 15(59), 467-487. https://doi.org/10.15366/rimcafd2015.59.005

Gutiérrez, O. (2006). Valoración del rendimiento táctico en balonmano a través de los coeficientes de eficacia. Aplicación del software SORTABAL v 1.0 (Tesis doctoral). Universidad Miguel Hernández, Elche.

Jiménez Jiménez, F. (2003). Construyendo escenarios, promoviendo aprendizajes: Las situaciones de enseñanza en la iniciación a los deportes de cooperación / oposición. En F. J. Castejón (Ed.), Iniciación deportiva. La enseñanza y el aprendizaje comprensivo en el deporte. Sevilla: Wanceulen.

Kelso, J. A. S. (2014). Coordination dynamics and cognition. En K. Davids, R. Hristovsky, D. Araújo, N. Balagué, C. Button, \& P. Passos (Eds.), Complex system in sport (pp. 18-43). London-NewYork: Routledge.

Lago, C. (2000). La acción motriz en los deportes de equipo de espacio común y participación simultánea. (Tesis doctoral). Universidade da Coruña, A 
Coruña.

Lasierra, G. (2017). Balonmano: la relación entre los sistemas de juego, los procedimientos colectivos y su eficacia (Tesis doctoral). Universitat de Lleida- INEFC-Lleida.

Lasierra, G., Serna, J., Salas, C., y López-Ros, V. (2019). Tactical assessment of team sports in a competitive context. An experience with University students. En C. Torrents \& E. Sebastiabi (Eds.). Sport, Physical Education and Performing Arts as tools of social transformation (Abstract book 30th FIEP world congress) (pp. 536-539). Barcelona: Inde.

López-Herrero, F., y Arias-Estero, J. L. (2019). Efecto de la modalidad de juego en baloncesto (5vs.5 y 3vs.3) sobre conductas motrices y psicológicas en alumnado de 9-11 años. Retos. Nuevas tendencias en Educación Física, Deporte y Recreación, 36, 345-361.

Lozano, D. (2014). Análisis del comportamiento táctico ofensivo en alto rendimiento en balonmano. (Tesis doctoral). Universitat de Lleida, Lleida.

Montoya, M. (2010). Análisis de las finalizaciones de los jugadores extremo en balonmano (Tesis doctoral). Universitat de Barcelona.

Morales-Belando, M., y Arias-Estero, J. L. (2015). Diferencias entre el juego 7 vs. 7 y el 4 vs. 4 en el balonmano escolar en relación al rendimiento, percepción del esfuerzo y la intencionalidad de práctica. Retos. Nuevas tendencias en Educación Física, Deporte y Recreación, 27, 34-39.

Neves, P. M. (2004). A «performance» diferencial em andebol (Tesis de maestría). Universidade do Porto, Porto.

Parlebas, P. (2008). Juegos, deporte y sociedades. Léxico de Praxiología Motriz. Barcelona: Paidotribo.

Pérez, S., Rodríguez, A., Sánchez, A., De Mena, J. M., Fuentes, J. M., Castaño, R., y Martín, N. (2019). Efecto de los juegos reducidos sobre jugadoras de fútbol. Revista internacional de medicina y ciencias de la actividad física y deporte, 19(74), 371-386. https://doi.org/10.15366/rimcafd2019.74.012

Rocha Santos, L. (2004). Tendências Evolutivas do Jogo de Andebol. Estudo centrado na análise da performance táctica de equipas finalistas em Campeonatos do Mundo e Jogos Olímpicos (Tesis doctoral). Universidade do Porto, Porto.

Rogulj, N., Srhoj, V., y Srhoj, L. (2004). The contribution of collective attack tactics in differentiating handball score efficiency. Collegium antropologicum, 28(2), 739-746.

Serra-Olivares, J., y García-López, L. M. (2016). Diseño y validación del test de conocimiento táctico ofensivo en fútbol (TCTOF). Revista internacional de medicina y ciencias de la actividad física y deporte, 16(63), 521-536. https://doi.org/10.15366/rimcafd2016.63.008

Sierra-Guzmán, R., Sierra-Guzmán, S., Sánchez, F., y Sánchez Sánchez, M. (2015). Análisis de las situaciones tácticas ofensivas de la selección española masculina de balonmano en desigualdad numérica en los campeonatos de Europa de Serbia 2012 y de Dinamarca 2014. Ebalonmano.com: Revista de Ciencias del Deporte, 11, 55-72.

Sousa, D. J., Prudente, J. F., Sequeira, P., López-López, J. A., y Hernández Mendo, A. (2015). Análisis de las situaciones de juego 2 vs2 en el campeonato europeo masculino de balonmano 2012: Aplicación de la técnica de coordenadas polares. Cuadernos de psicología del deporte, 
Rev.int.med.cienc.act.fís.deporte - vol. 20 - número 79 - ISSN: 1577-0354

15(1), 181-194. https://doi.org/10.4321/S1578-84232015000100018

Número de citas totales / Total references: $32(100 \%)$

Número de citas propias de la revista / Journal's own references: 4 (12,5\%)

Rev.int.med.cienc.act.fís.deporte - vol. 20 - número 79 - ISSN: 1577-0354 